\section{Catabolic plasmids, not dissimilatory}

SIR-A recent letter ${ }^{1}$ proposed that the term "dissimilatory plasmids" be used to describe the bacterial plasmids that encode pathways for the conversion of compounds such as toluene, naphthalene and camphor to central metabolites. Whilst agreeing with the authors that a single, generally accepted term for such plasmids should be used, I disagree with their solution of abandoning all three of the current terms in favour of a fourth.

Certainly 'degradative' does not necessarily imply the complete breakdown of the growth substrate and 'metabolic' could imply biosynthetic functions. However, it is my understanding that "catabolic' exactly covers the function of these plasmids, namely either the partial ${ }^{2}$ or the complete $^{3}$ breakdown of the growth substrates for the purposes of fuelling central metabolism. 'Dissimilatory' does not appear to supply any additional nuances of meaning, and suffers the double disadvantage of being difficult to enunciate from the lecture rostrum and subject to incorrect spelling, even by learned journals ${ }^{4}$.

\begin{tabular}{l} 
PetER A. Williams \\
Department of Biochemistry \& Soil \\
Science, \\
University College of North Wales, \\
Bangor, Gwynedd LL57 2UW, UK \\
1. Balajee, S., Boominathan, K. \& S. Mahadevan, A. Nature \\
719,728 (1986). \\
2. Fennewald, M., Benson, S., Oppici, M \& Shapiro, J. J. \\
Bact. 139, 940 (1979). \\
3. Worsey, M.J. \& Williams, P.A. J. Bact. 124, 7-13(1975). \\
4. Wheelis, M.L. A. Rev. Microbiol. 29, 505-524 (1975). \\
\hline
\end{tabular}

\section{Relationship of Blym genes to repeated sequences}

SIR-The Blym-1 gene of chicken was defined as a fragment of chicken lymphoma DNA which produced oncogenic transformation in mouse fibroblast cells ${ }^{1}$. From the DNA of mouse cells thus transformed, a clone of Blym- 1 was isolated, identified by its transforming activity, and sequenced $^{2}$. However, I now report that the sequence which was presented is actually a fragment from a very abundant dispersed repeat family in mouse DNA.

The repeat family is LINE-1 (ref. 3). Homology to "chicken Blym-1" was first discovered ${ }^{4}$ in a computer search with the distantly related human LINE-1 sequence, but is much more striking with the mouse LINE-1 sequence. The whole 590 nucleotides of "chicken Blym-1" aligns with nucleotides $2,402-2,995$ of the mouse sequence of ref. 5 , with only $10.5 \%$ mismatch (including 11 insertions or deletions of single nucleotides and one of four nucleotides). Over major parts of the sequence the mismatch is only $7.5 \%$. These values are typical for divergence between dispersed repeats within a single species. Indeed "chicken Blym-1" gave a very strong dispersed hybridization to mouse DNA on Southern blots 2 . Although some dispersed hybridization was also seen to chicken $\mathrm{DNA}^{2}$, its origin is unclear, as a similar mouse LINE-1 probe gave no hybridization to duck $\mathrm{DNA}^{6}$, which should be closely related to chicken DNA.

It is not evident how this LINE-1 fragment could have a phenotypic effect on mouse cells. The mouse genome already contains thousands of copies of such sequences. Although LINEs are derived from parental copies with two conserved open reading frames ${ }^{3,5}$, the "Blym-l" specimen has many frameshifts in the first of these and could only translate a fragment of the second. (With regard to the biology of mouse LINEs, it is interesting that the divergence of the "Blym-1" specimen from the specimen of ref. 5 is greatest at and around the junction between the two LINE-1 open reading frames.)

A human "Blym-1 gene" was cloned by similar methods ${ }^{7}$. Homology was claimed between the chicken and human "Blym-1" genes, but it required many gaps and is not substantiated by diagonal matrix plots of the DNA sequences (not shown). "Human Blym-1" is unrelated to LINE-1 sequences, but the central part of it is a human Alu dispersed repeat, only 50 nucleotides of which were originally noticed ${ }^{7}$. This repeat covers nucleotides $262-567$, with $74 \%$ homology to the consensus of ref. 8 , and it substantially overlaps the proposed Blym- 1 open reading frame. Since Alu repeat sequences are species-specific, the significance of this gene also seems questionable.

\section{MRC Laboratory of Molecular Biology,} Hills Road, Cambridge CB2 2QH, UK

\footnotetext{
. Cooper, G.M. \& Neiman, P.E. Nature 287, 656-659 (1980) 2. Goubin. G. et al. Nature 302, 114-119 (1983)

3. Singer, M.F. \& Skowronski, J. Trends biochem. Sci. 10 119-122 (1985).

Hattori, M. Hidaka, S. \& Sakaki, Y. Nucleic Acids Res. 13, 7813-7827 (1985)

Loeb, D.D. et al. Molec. cell. Biol. 6, 168-182 (1986)

6. Burton, F.H. et al. J. molec. Biol. 187, 291 (1986).

7. Diamond, A., Devine, J.M. \& Cooper, G.M. Science 225. $516-519(1984)$

8. Deininger, P.L. et al. J. molec. Biol. 151, 17-33 (1981).
}

SIR-The molecular clone designated chicken Blym- 1 was isolated from NIH 3T3 mouse cells transformed by DNA of a chicken B-cell lymphoma using sibselection to identify a clone which induced transformation of NIH 3T3 cells upon transfection ${ }^{1}$. The cloned transforming gene hybridized to highly repeated sequences in mouse DNA and to a small family of related sequences in DNAs of chicken and human cells ${ }^{1}$. Its nucleotide sequence was not closely related to any published sequences, although the predicted coding sequence was partially homologous to transferrins ${ }^{1}$. However, the transforming gene contained two short regions ( 20 nucleotides) which were $70-$ $80 \%$ homologous to mouse satellite DNA and which were thought to account for the hybridization to repetitive mouse sequen$\operatorname{ces}^{1}$. Therefore, the hybridization data did not determine whether the gene was of chicken or mouse origin ${ }^{1}$. However, we considered it likely that the gene represented the transforming gene of chicken bursal lymphomas since serial transmission of transforming genes was established as a high-frequency event whereas activation of the transforming potential of normal cell genes was infrequent ${ }^{2}$ and preliminary data indicated expression of homologous RNAs and a candidate protein product in chicken lymphoma as well as mouse cells.

Hattori et al. ${ }^{3}$ recently determined the complete sequence of a full-length member (T $\beta$ G41) of the human long interspersed repetitive sequence family (LINES) and found that the T $\beta$ G41 sequence was $60 \%$ homologous to that of chicken Blym-1. In addition, the aminoacid sequence predicted for the open reading frame of $T \beta G 41$ was siginficantly homologous to transferrin ${ }^{3}$.

Loeb et al ${ }^{4}$ have now reported the complete sequence of the $\mathrm{A} 2$ member of the mouse LINES family. The A2 sequence contains a region (nucleotides 2,4022,990 ) which is $90 \%$ homologous to chicken Blym-1. These data indicate that chicken Blym-1 is closely related to LINES and suggest that chicken Blym-I may represent a portion of a mouse LINES element whose transforming potential was activated during the process of molecular cloning from transformed NIH cells. Its activation might then be a consequence of truncation or rearrangement, as has been demonstrated for other transforming sequences ${ }^{5-7}$.

To resolve the origin of chicken Blym-1, an oligonucleotide corresponding to positions $242-287$ of the chicken Blym-1 sequence $^{1}$ was synthesized and used as a probe for blot-hybridization analysis. This probe hybridized to mouse repetitive sequences but did not detect a homologous chicken sequence, although it hybridized to reconstruction digests containing the equivalent of one copy per genome of the chicken Blym-1 clone. We therefore conclude that the transforming gene designated chicken Blym-1 is a portion of a mouse LINES element whose transforming potential was probably activated during molecular cloning rather than the transforming gene originally detected by transfection of chicken bursal lymphoma DNAs ${ }^{8}$. We propose that the gene be redesignated mouse LINES transforming element (LINES-TE).

The mouse LINES-TE was used as a probe to isolate related sequences from a 